OPEN ACCESS

Edited by:

Gedis Grudzinskas,

Independent researcher, London,

United Kingdom

Reviewed by:

Giuseppe Morgante,

University of Siena, Italy

Giuliano Marchetti Bedoschi,

University of São Paulo, Brazil

Zhiqin Bu,

Zhengzhou University, China

${ }^{*}$ Correspondence:

Human M. Fatemi

human.fatemi@fatemifertility.com

Specialty section:

This article was submitted to

Reproduction,

a section of the journa

Frontiers in Endocrinology

Received: 17 September 2021

Accepted: 03 January 2022

Published: 09 February 2022

Citation:

Ghasemi M, Aleyasin A, Fatemi HM, Ghaemdoust $F$ and Shahrakipour M (2022) Uterine Cavity Irrigation With Office Hysteroscopy During Ovarian Stimulation for IVF: A Randomized Controlled Trial.

Front. Endocrinol. 13:778988. doi: 10.3389/fendo.2022.778988

\section{Uterine Cavity Irrigation With Office Hysteroscopy During Ovarian Stimulation for IVF: A Randomized Controlled Trial}

\author{
Marzieh Ghasemi ${ }^{1}$, Ashraf Aleyasin ${ }^{2}$, Human M. Fatemi ${ }^{3 *}$, Faezeh Ghaemdoust ${ }^{4}$ \\ and Mahnaz Shahrakipour ${ }^{5}$ \\ 1 Pregnancy Health Research Center, Department of Obstetrics and Gynecology, Zahedan University of Medical Sciences, \\ Zahedan, Iran, ${ }^{2}$ Department of Infertility, Faculty of Medicine, Shariati Hospital, Tehran University of Medical Sciences, \\ Tehran, Iran, ${ }^{3}$ In-Vitro-Fertilisation (IVF) Department, Assisted Reproductive Technology (ART) Fertility Clinics, Abu Dhabi, \\ United Arab Emirates, Clinical Laboratory, Assisted Reproductive Technology (ART) Fertility Clinics, Abu Dhabi, United Arab \\ Emirates, ${ }^{4}$ School of Medicine, Tehran University of Medical Sciences, Tehran, Iran, ${ }^{5}$ Department of Biostatistics, Zahedan \\ University of Medical Sciences, Zahedan, Iran
}

Objective: This was a non-blinded randomized controlled study to evaluate whether endometrial irrigation via office hysteroscopy during the early follicular phase would lead to a higher level of live birth rates compared to no irrigation in the fresh embryo transfer cycle.

Method: The study was conducted in Tehran university of medical sciences from June 2015 to June 2016. women under the age of 40 with primary infertility without history of previous IVF/ICSI or hysteroscopic examination, were included. Controlled ovarian hyperstimulation was done. Hysteroscopy was performed in the early mid-follicular phase of a stimulation cycle (day 5-7) with a vaginoscopy approach and saline irrigation in hysteroscopy group. Embryo-transfer was done in the same cycle.

Results: 228 patients completed their participation in the study. In the fresh cycle, clinical pregnancy rate was $46 \%$ in the hysteroscopy group and $40.43 \%$ in the control group. (pvalue $=0.326, R R=1.16$ [95\% Cl: 0.862 to 1.56 ]). Live birth rate was $41.28 \%$ in the hysteroscopic group and 31.93\% in the control group ( $p$-value $=0.143, R R=1.293$ [95\% Cl: 0.916 to $1.825 \mathrm{~J}$ ). For those patients having surplus cryopreserved embryos, after 2 months, a second embryo transfer was performed. The cumulative LBR was $44.05 \%$ in the hysteroscopic group and $32.25 \%$ in the control group ( $p$-value $=0.029, R R=1.368$ [95\% Cl: 1.031 to 1.815 ], $R D=11.9 \%$ [95\% Cl: $1.2 \%$ to $22.3 \%$ ] and $N N T=8$ [95\% Cl: 4 to 85]).

Conclusion: The current study clearly demonstrated a significantly higher cumulative live birth rate in the intervention group.

Clinical Trial Registration: [https://www.irct.ir/trial/19586], identifier IRCT20 16011022795 N2.

Keywords: IVF cycle, uterine cavity irrigation, office hysteroscopy, endometrial receptivity, implantation 


\section{INTRODUCTION}

Notwithstanding the many recent advances in the field of ART, the chance of success is still very limited. Based on various publications, the implantation rate per embryo transfer is still around 35\% (Planned transfers of cleavage-stage embryos: competency $\geq 25 \%$; benchmark $\geq 35 \%$ - Blastocyst transfers: competency $\geq 35 \%$; benchmark $\geq 60 \%$ ) (1).

For a pregnancy to occur, a receptive endometrium, a functional embryo at blastocyst developmental stage and synchrony between the embryo and the endometrium is required (2). Failure to achieve receptivity and synchrony results in infertility and is a limiting factor for success in IVF treatment.

Among the papers and studies focusing on endometrial receptivity at the time of embryo transfer, Endometrial scratching in the cycle preceding IVF had been regarded as one of the most noteworthy methods that can affect the endometrial receptivity and probably improve the implantation rate (3). Although there are many explanations for the possible effect of endometrial injury on implantation, latest rigorous clinical trials and meta-analyses have claimed the inefficiency of this procedure on the implantation rate $(4,5)$. However, because of manifold methods and different timings and anatomical locations of the injury caused, it is still not completely evident whether any manipulation at any specific time of the cycle can improve the implantation results (6).

Alongside with endometrial scratching in the recent years, office hysteroscopy has been represented by many researchers as a means of improving uterine receptivity. Some successful outcomes have indeed been obtained through finding pathologies via hysteroscopy and treating them (7-9).

The current results also suggested that the benefit of hysteroscopy could extend beyond correction of uterine pathology. Some studies demonstrated that irrigation of the uterine cavity might even change the uterine environment and have a positive impact on uterine receptivity and the following implantations as well (10-12). Moreover, OH (Office Hysteroscopy), by dilatating the cervix would facilitating the subsequent embryo transfer and possibly by a total endometrial irrigation, create an aseptic endometrial inflammation (7).

Most of the aforementioned studies evaluated different aspects of using hysteroscopy or scratching and their effect on implantation. However, there are scarce data to date on the value of irrigation with hysteroscopy during the first IVF attempt. The aim of this study is to verify the role of uterine irrigation through hysteroscopy during the follicular phase, aiming to improve IVF outcomes in first IVF cycle candidates.

\section{MATERIALS AND METHODS}

The present study was conducted from June 2015 to June 2016 in the IVF Centre of Shariati hospital, Tehran University of Medical Sciences. It is a non-blinded randomized controlled trial which aimed to assess the possible benefit of hysteroscopic uterine irrigation, prior first IVF cycle, on the reproductive outcomes.
The study protocol was approved by the IRCT and ethics committee of Tehran University of Medical Sciences (Ethics committee reference number: IR.TUMS.REC.1394.1598). Trial registration number: IRCT2016011022795N2

\section{Participants}

248 patients with primary infertility were recruited for this study. Participants with regular menstrual cycle (regular menses defined as a duration of 24-35 days), age $\leq 40$ years, BMI between 19- 30, without any prior hysteroscopic examination or previous IVF or ICSI, normal Transvaginal sonography (TVS) in the last month and hysterosalpingography (HSG) (between 6-24 months), who were scheduled for the first IVF cycle, were included.

Exclusion criteria were determined as recurrent miscarriage (3 or more miscarriages), intermenstrual bleeding, any doubt about uterine cavity abnormality, azoospermia, patients with poly cystic ovary syndrome (PCOS) or endometriosis AFS 3/4, hydrosalpinx, ovarian cysts and cancellation of the same cycle for any reason. Moreover, participants were excluded, if there were any difficulties with the hysteroscopy procedure, such as bleeding post procedure or detecting uterine cavity abnormalities or inability to perform the procedure (and necessity to use Hegar dilator in order to dilatated the cervix (Figure 1).

Prior entering the study, the purpose and process of the study was clearly explained to all participants and they all gave written informed consent. The patients had the right to quit the study at any time for any reason. Physicians and other healthcare workers involved in this study were all respectful to the principles of Good Clinical Practice and the Declaration of Helsinki.

All patients participating in the study underwent the standard infertility work-ups, consisting of the medical history, physical examination, hormonal status and their spouses' semen analysis.

The patients who accepted to join the study and met all the relevant criteria, were randomized into two groups by a web-based randomization program which was based on block randomization: group I- with hysteroscopic irrigation in the IVF cycle (intervention) and group II-without hysteroscopy (control).

\section{Procedures \\ Ovarian Hyper Stimulation and Embryo Transfer Methods}

Controlled ovarian hyper stimulation was done in an antagonist regimen in the two groups with recombinant FSH (Gonal-f, Merck-Sereno) starting on day 2 of the cycle at a dose of 150 IU daily for the first 6 days, the dosage was adjusted according to the patient's individual ovarian response. The $\mathrm{GnRH}$ antagonist (cetrorelix, Actoverco) was initiated when the leading follicles had a diameter $14 \mathrm{~mm}$. The final oocyte maturation was achieved by the administration of HCG (choragun) I.M. 10000 IU as soon as 3 follicles reached $17 \mathrm{~mm}$ in diameter. Oocyte retrieval was carried out 36 hours post HCG administrations. Embryo transfer was performed on day 3 after oocyte retrieval and only two grade 1 embryos (cleavage) were transferred. Luteal phase supplementation consisted of suppository progesterone (supp Cyclogest, Actoverco $400 \mathrm{mg}$ BID) after oocyte retrieval and was continued till the 8 th week of gestation. 


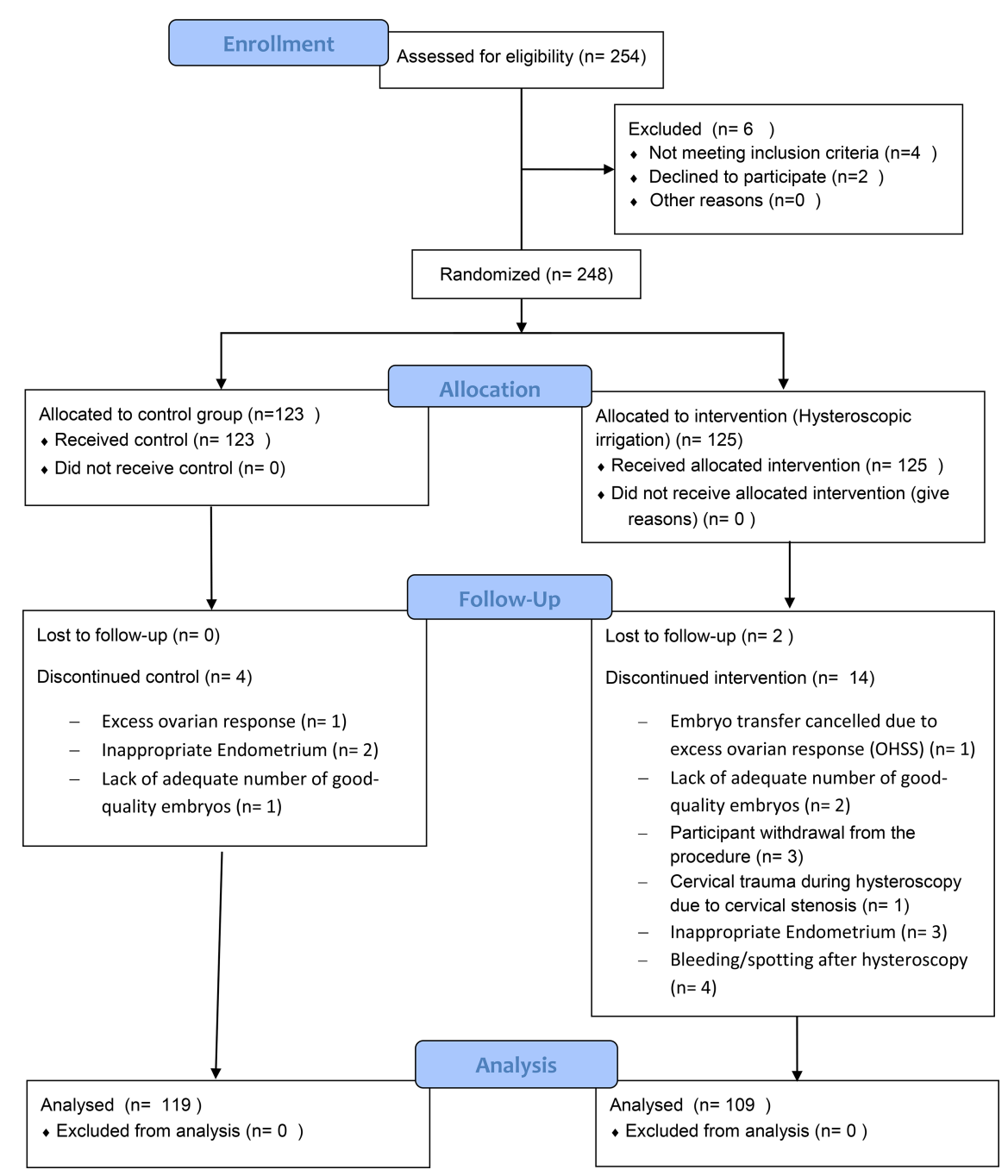

FIGURE 1 | CONSORT flow diagram of the study.

\section{Hysteroscopy}

Hysteroscopy and irrigation of uterine cavity with a large amount of saline solution (200-300 cc) was perform for the intervention group in the early mid-follicular phase of a stimulation cycle (day 5-7). It was implemented with a five French working channel and a $30^{\circ}$ direction of view hysteroscope with a Vaginoscopic approach. The endocervical canal, uterine cavity, tubal orifices and endometrium were all inspected and recorded on standardized forms. In order to reduce inter-operator bias, all hysteroscopies were conducted by the same surgeon. Finally, participants in both groups underwent embryo transfer in the same cycle as the hysteroscopic irrigation (for intervention group).

\section{Embryo Transfer}

Morphology of the embryos were categorized as grade 1 based on Giorgetti-4-point-score (13). Embryo transfer for all participants were implemented after 3 days of fertilization based on standard protocols (14).
After 14 days of embryo transfer, beta-HCG was tested and if positive results were reported, ultrasonography was performed in order to confirm the clinical pregnancy. In case of pregnancy failure and existence of frozen embryos, they would be included for second transfer cycle.

\section{Inclusion Criteria to the Second Freezing Cycle}

Workups for the second cycle started for participants who did not get pregnant after the embryo transfer: OCP was prescribed in the next menstrual cycle. After 5 days of OCP free interval the cycle, estradiol valerate $6 \mathrm{mg} /$ day (Aburaihanco $2 \mathrm{mg}$ Tab) was administered for 10 days. At this point, if the endometrial thickness (ET)was not proper for the procedure (ET less than $8 \mathrm{~mm})$, dose-adjustment was done ( $8 \mathrm{mg}$ ) and another ultrasonography was performed 3-5 days later to evaluate whether the ET is ready (If the endometrium did not reach that level, the participants would be excluded from the study). 
Once the endometrial thickness reached $8 \mathrm{~mm}$ or more, progesterone was prescribed (supp Cyclogest, Actoverco 400 mg BID) and embryo transfer would start after completing the 3 days of progesterone administration (in the $4^{\text {th }}$ day). Embryo transfer was done by using COOK catheter. After 14 days of embryo transfer, beta-HCG was tested and if positive results were reported, ultrasonography was performed in order to confirm the clinical pregnancy in 6 weeks.

\section{Outcome Measures}

The primary objective of the current study was live birth rate (LBR). Secondary outcomes were clinical pregnancy rate, Chemical pregnancy rate, Abortion rate and implantation rate (per cycle and per transfer). Pregnancy test was done 2 weeks after embryo transfer and positive tests were confirmed after a week. The aforementioned outcomes are calculated as indicated in the following section.

\section{Definitions}

Chemical (biochemical) pregnancy rate: beta-HCG serum level more than $25 \mathrm{mIU} / \mathrm{ml}$ in 2 weeks after embryo transfer

Clinical pregnancy rate: observing one or more gestational sacs around the age of 6 weeks by Ultrasound imaging

Live birth rate: any parturition after 27 weeks

Abortion rate: any pregnancy failure before the 20 weeks.

Implantation rate: number of gestational sacs observed divided in the number of embryos transferred

\section{Statistical Analysis}

Sample size was calculated based on the observed IVF cycle outcomes and differences in outcomes from existing literature. Based on a study by Rama Raju et al. (15), clinical pregnancy rate considered $25 \%$ in control group and $40 \%$ in $\mathrm{OH}$ group. For the difference of $15 \%$ increase in CPR and a power of $80 \%$ and a one sided alpha error of 5\%, 240 participants in total were calculated to be included. Considering the lost to follow-up in some patients, 254 women enrolled in the study statistical analysis was performed using the SPSS version 22 and web based EBM calculator.

Primary transfer results and secondary (freezing) transfer results were calculated separately. Thereafter, all transfers underwent a cumulative analysis which were evaluated both per transfer and per cycle.
In order to make sure that the randomization was properly implemented, patients and their ovulation induction characteristics, T-test or its non-parametric equivalent were done.

For comparing the pregnancy outcomes between the two groups chi-square test was applied. Relative Risk with 95\% CI were also calculated. For statistical significance, P-value $<0.05$ was considered.

\section{RESULTS}

From 254 patients whose eligibility were assessed, 248 enrolled in the study and were randomly assigned to any of the intervention or control group (125 participants in Hysteroscopy group and 123 participants in control group). From the 125 participants in the hysteroscopy group, 16 were excluded due to different reasons and finally 109 participants from this group underwent embryo transfer. From 123 participants in control group, 4 were excluded and 119 participants underwent embryo transfer (Figure 1).

\section{Participant's Characteristics}

Relevant characteristics of participants were compared between these two groups. There were no significant differences in the Age, Weight, BMI and ovarian reserve (tested by FSH, AMH, AFC) between the two groups (Table 1).

\section{Cycle's Characteristics and First Transfer Features}

Characteristics of ovarian stimulation cycles were also compared between these 2 groups. Variables including duration of ovarian stimulation, gonadotropin dosage, number of follicles with 14 $\mathrm{mm}$ or more in diameter in the day of final oocyte maturation, number of retrieved oocytes, number of metaphase II oocytes and also number of yielded embryos were all compared. Number of $\mathrm{GnRH}$ antagonist administration. There were no significant difference between the 2 groups compared which indicates the similarity of baseline characteristics in both groups. Moreover, the number of transferred embryos, number of frozen embryos and the endometrial thickness did not reveal any significant difference between the two groups compared (Tables 2A, B).

\section{Fresh Embryo Transfer Results}

In the fresh embryo transfer of the hysteroscopy group, 53\% of the included patients had a positive beta-hCG as compared to $45.5 \%$ in the control group ( $p$-value $=0.23, R R=1.173$ [95\%CI: 0.9 to 1.527$]$ ).

TABLE 1 | Patients' Characteristics.

\begin{tabular}{|c|c|c|c|}
\hline Variable & Intervention group (mean $\pm S D$ ) & Control group (mean \pm SD) & p-value \\
\hline Age & $29.90 \pm 3.805$ & $30.54 \pm 4.20$ & 0.233 \\
\hline Weight & $63.84 \pm 9.09$ & $61.99 \pm 8.97$ & 0.115 \\
\hline FSH & $4.52 \pm 2.17$ & $5.03 \pm 2.32$ & 0.094 \\
\hline $\mathrm{LH}$ & $7.73 \pm 4.78$ & $7.57 \pm 4.84$ & 0.797 \\
\hline $\mathrm{AMH}$ & $2.97 \pm 1.22$ & $3.37 \pm 2.84$ & 0.154 \\
\hline AFC & $10.81 \pm 2.67$ & $10.68 \pm 2.58$ & o. 709 \\
\hline TSH & $2.03 \pm 0.91$ & $1.97 \pm 1.01$ & 0.637 \\
\hline $\mathrm{BMl}$ & $24.19 \pm 2.84$ & $23.94 \pm 3.52$ & 0.545 \\
\hline
\end{tabular}


TABLE 2 | A) Cycles' characteristics B) first transfer characteristics.

\begin{tabular}{|c|c|c|c|}
\hline Variable & Intervention group (mean $\pm S D$ ) & Control group (mean $\pm S D$ ) & p-value \\
\hline \multicolumn{4}{|l|}{ A) Cycle's Characteristics } \\
\hline Duration of ovulation induction (day) & $10.15 \pm 0.812$ & $10.25 \pm 1.04$ & 0.392 \\
\hline Gonadotropin dosage & $2740.43 \pm 638.25$ & $2877.75 \pm 724.50$ & 0.052 \\
\hline Number of folicles $\geq 14 \mathrm{~mm}$ & $12.58 \pm 4.57$ & $12.16 \pm 3.68$ & 0.428 \\
\hline Number of oocytes after pick up & $12.88 \pm 4.72$ & $12.26 \pm 5.12$ & 0.327 \\
\hline Number of oocytes in Metaphase ॥ & $8.82 \pm 4.36$ & $8.56 \pm 4.67$ & 0.657 \\
\hline \multicolumn{4}{|l|}{ B) First transfer characteristics } \\
\hline Total Embryo & $7.73 \pm 4.07$ & $7.29 \pm 4.16$ & 0.407 \\
\hline Number of embryo transferred & $2.33 \pm 0.90$ & $2.44 \pm 0.79$ & 0.292 \\
\hline Number of embryo freezed & $4.89 \pm 3.84$ & $4.54 \pm 4.01$ & 0.489 \\
\hline Endometrial thickness & $8.18 \pm 0.71$ & $8.23 \pm 0.71$ & 0.622 \\
\hline
\end{tabular}

Clinical pregnancy rates have been $46 \%$ and $40.33 \%$ in the hysteroscopy and control group, respectively (p-value $=0.32, R R=$ 1.16 [95\%CI: 0.862 to 1.56$]$ ).

Live birth rates have been $41.28 \%$ in hysteroscopy and $31.93 \%$ in control group, respectively ( $p$-value $=0.143, R R=1.293$ [95\%CI: 0.916 to 1.825$]$ )

From 58 patients who got pregnant in the hysteroscopic irrigation group, 45 resulted in live births (41\%) with an abortion rate of $22.41 \%$. In the control group, out of 54 women who got pregnant, 38 of them had live births (31\%), with an abortion rate of $25.92 \%$. (p-value: 0.664 )

The implantation rates were $22.85 \%$ and $18.62 \%$ in hysteroscopy ad control groups, respectively (p-value: 0.206 ).

While none of the outcome measures related to fresh embryo transfer were statistically significant, 9.4\% difference was showed in live birth rates between the two groups compared, in favour of the hysteroscopy group (Table 3)

\section{Second Transfer Characteristics}

Among participants, who did not get pregnant in the first transfer and had frozen embryos available, underwent a subsequent cryo-embryo transfer.

Characteristics for this second transfer are presented in Table 4. Variables like number of embryos transferred, endometrial thickness, day of embryo transfer were compared and did not reveal any significant differences.

\section{Frozen Embryo Transfer Results}

34 participants from hysteroscopy group and 58 participants from the control group underwent a second embryo transfer procedure using frozen embryos. From the 34 patients in the hysteroscopy group, 21 had positive pregnancy test $(61.76 \%)$ and in the control group, from the 58 participants, 25 (43.10\%) had positive beta-hCG ( $p$-value $=0.084, R R=1.433$ [95\%CI: 0.964 to 2.131$]$ ).
Clinical pregnancy rate was $52.94 \%$ and $36.20 \%$ in the hysteroscopy and control group, respectively ( $p$-value $=0.12$, $R R=1.462$ [95\%CI: 0.918 to 2.33$]$ ).

Live birth rate had an average of $52.94 \%$ in the hysteroscopy group and $32.75 \%$ in the control group ( $\mathrm{p}$-value $=0.06, R R=1.616$ [95\%CI: 0.994 to 2.628 ]).

Abortion rate was $14.28 \%$ and $24 \%$ in the intervention and control group, respectively ( $\mathrm{p}$-value $=0.40$ ).

Implantation rate in hysteroscopy group was $18.62 \%$ compared to $16.12 \%$ in the control group ( $p$-value $=0.117$ ).

Similar to the first transfer, none of the outcome measures in the second transfer were significantly different between 2 groups. However, the live birth rate was $20 \%$ higher in the intervention group. Detailed results of this second transfer are presented in Table 5.

\section{Cumulative Results}

Cumulatively (Table 6), 143 and 177 transfers were conducted for the hysteroscopy and control group, respectively. Cumulative positive pregnancy rate was $55.24 \%$ in the hysteroscopy group and $44.63 \%$ in the control group ( $p$-value $=0.050, R R=1.238$ [95\%CI: 0.993 to 1.543$]$ ) Clinical pregnancy in hysteroscopy group was $48.25 \%$ and in control group 38.98\% ( $p$-value $=0.096, R R=1.238$ [95\%CI: 0.963 to 1.59$]$ ). Live birth rates were $44.05 \%$ and $32.20 \%$ in the hysteroscopy and control group, respectively ( $p$-value $=0.029, R R=1.368$ [95\%CI: 1.031 to 1.815$]$ ). The difference in live birth rate between two groups was statistically significant in favour of the hysteroscopy group (live birth risk difference: $11.9 \%$ [95\% CI: $1.2 \%$ to $22.3 \%$ ]).

Abortion rates were $20.25 \%$ in the intervention group and $25.31 \%$ in the control group ( $\mathrm{p}$-value $=0.448$ ). Implantation rate in the hysteroscopy group was $23.22 \%$ as compared to $17.78 \%$ in the control group ( $\mathrm{p}$-value $=0.05)$.

Cumulatively (Table 6), chemical pregnancy rate was $72.47 \%$ among the hysteroscopy group and $66.38 \%$ in the control group ( $p$ value $=0.394, R R=1.092$ [95\%CI: 0.919 to 1.297$]$ ). Clinical pregnancy

TABLE 3 | Fresh Embryo Transfer results.

\begin{tabular}{|c|c|c|c|c|}
\hline Variable & Intervention group (109) & Control group (119) & p-value & $\mathrm{RR}[95 \% \mathrm{Cl}]$ \\
\hline Chemical Pregnancy Rate & 58 (53\%) & $54(45.4 \%)$ & 0.237 & $1.173[0.9$ to 1.527$]$ \\
\hline Clinical Pregnancy Rate & $51(46 \%)$ & 48 (40.33\%) & 0.326 & $1.16[0.862$ to 1.56$]$ \\
\hline Live Birth Rate & 45 (41.28\%) & 38 (31.93\%) & 0.143 & $1.293[0.916$ to 1.825 \\
\hline
\end{tabular}

live birth risk difference: $9.4 \%$ [95\% Cl: $-3.1 \%$ to $21.5 \%$ ]. 
TABLE 4 | Second (Frozen) Transfer Characteristics.

\begin{tabular}{|c|c|c|c|}
\hline Variable & Intervention group (mean $\pm S D)$ & Control group (mean $\pm S D$ ) & p-value \\
\hline Number of (frozen) embryos transferred & $2.52 \pm 0.50$ & $2.67 \pm 0.47$ & 0.176 \\
\hline Endometrial thickness & $8.23 \pm 0.424$ & $8.28 \pm 0.84$ & 0.755 \\
\hline
\end{tabular}

TABLE 5 | Frozen Embryo Transfer Results.

\begin{tabular}{|c|c|c|c|c|}
\hline Variable & Intervention group (34) & Control group (58) & p-value & RR $[95 \% \mathrm{Cl}]$ \\
\hline Chemical Pregnancy Rate & $21(61.76 \%)$ & 25 (43.10\%) & 0.084 & 1.433 [0.964 to 2.131] \\
\hline Clinical Pregnancy Rate & 18 (52.94\%) & $21(36.20 \%)$ & 0.117 & $1.462[0.918$ to 2.33$]$ \\
\hline Live Birth Rate & 18 (52.94\%) & 19 (32.75\%) & 0.06 & 1.616 [0.994 to 2.628] \\
\hline
\end{tabular}

live birth risk difference: $20.2 \%$ [95\%Cl: $-0.5 \%$ to $39.1 \%$ ].

TABLE 6 | Cumulative Pregnancy Outcomes.

\begin{tabular}{|c|c|c|c|c|c|}
\hline A) per transfer & Variable & Intervention group (143) & Control group (177) & p-value & RR $(95 \% \mathrm{Cl})$ \\
\hline & Chemical Pregnancy Rate & 79 (55.24\%) & 79 (44.63\%) & 0.05 & $1.238[0.993$ to 1.543$]$ \\
\hline & Clinical Pregnancy Rate & 69 (48.25\%) & 69 (38.98\%) & 0.096 & 1.238 [0.963 to 1.59$]$ \\
\hline & Live Birth Rate & $63(44.05 \%)$ & $57(32.25 \%)$ & 0.029 & $1.368[1.031$ to 1.815$]$ \\
\hline \multirow[t]{4}{*}{ B) per cycle (patient) } & Variable & Intervention group (109) & Control group (119) & p-value & RR $(95 \% \mathrm{Cl})$ \\
\hline & Chemical Pregnancy Rate & 79 (72.47\%) & 79 (66.38\%) & 0.394 & $1.092[0.919$ to 1.297$]$ \\
\hline & Clinical Pregnancy Rate & $69(63.3 \%)$ & 69 (57.98\%) & 0.493 & $1.092[0.886$ to 1.346$]$ \\
\hline & Live Birth Rate & $63(57.79 \%)$ & 57 (47.9\%) & 0.173 & $1.207[0.943$ to 1.544$]$ \\
\hline
\end{tabular}

in hysteroscopy group was $63.3 \%$ and in control group was about 57.98\% ( $p$-value $=0.493, R R=1.092$ [95\%CI: 0.886 to 1.346$]$ ). Live birth rates were $57.79 \%$ and $47.9 \%$ in the hysteroscopy and control group respectively ( $p$-value $=0.173, R R=1.207$ [95\%CI: 0.943 to $1.544])$. Neither of the per cycle results were statistically significant. However, live birth rate was $9.9 \%$ higher in the intervention group (live birth risk difference: 9.9\% [95\%CI: -3\% to $22.4 \%]$ ).

\section{DISCUSSION}

In this study, hysteroscopic irrigation did not show a significant improvement of the Live birth rate in the fresh cycle $(R R=1.293$ [95\%CI: 0.916 to 1.825$]$ ]). However, taking the frozen cycle into account, this intervention clearly demonstrated a significantly higher cumulative live birth rate $(R R=1.368$ [95\%CI: 1.031 to 1.815]. despite being significant, since the sample size was not calculated regarding the cumulative results, we can not claim this significance to be important. However, these results might help in hypothesizing and conducting more rigorous similar studies with greater sample sizes and these future study and metaanalyses might show a noteworthy association.Moreover, the current study demonstrated that an atraumatic hysteroscopy during ovarian stimulation for IVF, doesn't not harm the patient and significantly increases the cumulative livebirth rate

In the past, Endometrial Scratching (ES) was one of the most popular methods proposed to possibly improve the endometrial receptivity. However, recent meta-analysis and high-powered studies failed to demonstrate any benefit of ES on pregnancy outcome. $(4,5,16,17)$. The most notable explanation is that ES could be highly traumatic and might damage some areas of endometrium. However, this is not the case with hysteroscopy and irrigation. In the current study, no harm/trauma was caused to the endometrium. The uterine cavity was accessed just by the saline without any scratching or biopsy. We did so since it was presumed that taking a piece of endometrium might cause damage to the endometrium $(4,18)$ and might later lead to a disturbance for the implantation in the next transfer cycle. Karimzadeh et al. reported that performing ES in the same transfer cycle at the day of oocyte retrieval would have harmful effects on the outcomes (18).

Aside from being harmless, there are other hypothesis supporting the possible benefits of cavity irrigation: First, irrigation can mechanically remove detrimental anti-adhesive glycoproteins from the surface of the endometrium and subsequently improve the endometrial receptivity (11). Considering that hysterosalpingography can sometimes improve the pregnancy outcomes, a growing body of evidence supports the hypothesis that uterine flushing can improve fertilization by removing debris from tubes and also changing the production of cytokines (19).

Another explanation emphasizes the act of hysteroscopy itself as a diagnostic procedure; crossing the cervical canal with the tip of hysteroscope might lead to the lysis of cervical adhesions and might help in gathering information about the morphology of the cervical canal. These factors can facilitate the upcoming embryo transfer procedure $(3,20,21)$.

Another plausible explanation suggested on all kinds of mechanical manipulations is that those acts can initiate changes in the immune system and gene expression in such a direction that could help to improve receptivity and 
implantation $(20,21)$. Inagaki et al. showed that in patients with recurrent implantation failure the level of MMP activity and cytokine concentrations had a different pattern in the lavage (22); the effect of irrigation on immunity-related factors and cytokines can probably help in reversing this pattern.

Moreover, it was also suggested that mechanical manipulation in the preceding cycle can be more effective since these changes require time in order to show the results and also intervention in the same cycle as embryo transfer can disturb the endometrium (21).

Theoretically, fluid infusion and irrigation of the uterine cavity could also be considered as a form of an "atraumatic global endometrial injury". Some studies suggested the potential effect of immunomodulation triggered by uterine bathing using Lipiodol ${ }^{\circledR}$ $(23,24)$. In contrast, some other trials found no difference in using pharmacologically neutral gels for uterine bathing $(25,26)$

Salehpour et al. showed in their trial that intrauterine saline infusion using IUI catheter during IVF cycles could have a negative impact on pregnancy outcomes in RIF patients (27).

Throughout our study, this hysteroscopic irrigation method was well-tolerated and accepted by the patients in the intervention group. No case of endometritis was found.

Lack of blinding and small sample size were among the limitations of our study, given that an extra procedure like hysteroscopy might encourage the intervention group's participants to cooperate more widely than the other group's.

In conclusion, the current study could not show a significant difference in any of the pregnancy outcomes between the groups. Although there was a significantly higher cumulative per transfer live birth rate in the intervention group. Future, large RCTs are required to confirm the current findings.

\section{REFERENCES}

1. ESHRE Special Interest Group of Embryology and Alpha Scientists in Reproductive Medicine. The Vienna Consensus: Report of an Expert Meeting on the Development of ART Laboratory Performance Indicators. Reprod BioMed Online (2017) 35(5):494-510. doi: 10.1016/j.rbmo. 2017.06.015

2. Simón C, Martín JC, Pellicer A. Paracrine Regulators of Implantation. Baillieres Best Pract Res Clin Obstet Gynaecol (2000) 14(5):815-26. doi: 10.1053/beog.2000.0121

3. El-Toukhy T, Sunkara S, Khalaf Y. Local Endometrial Injury and IVF Outcome: A Systematic Review and Meta-Analysis. Reprod BioMed Online (2012) 25(4):345-54. doi: 10.1016/j.rbmo.2012.06.012

4. Frantz S, Parinaud J, Kret M, Rocher-Escriva G, Papaxanthos-Roche A, Creux H, et al. Decrease in Pregnancy Rate After Endometrial Scratch in Women Undergoing a First or Second In Vitro Fertilization. A Multicenter Randomized Controlled Trial. Hum Reprod (2019) 34(1):92-9. doi: 10.1093/humrep/dey334

5. Lensen S, Osavlyuk D, Armstrong S, Stadelmann C, Hennes A, Napier E, et al. A Randomized Trial of Endometrial Scratching Before In Vitro Fertilization. N Engl J Med (2019) 380(4):325-34. doi: 10.1056/NEJMoa1808737

6. Odendaal J, Quenby S. A Randomized Trial of Endometrial Scratching Before In Vitro Fertilization. N Engl J Med (2019) 380(18):1777. doi: 10.1056/ NEJMc1902642

7. Bosteels J, Weyers S, Puttemans P, Panayotidis C, Van Herendael B, Gomel V, et al. The Effectiveness of Hysteroscopy in Improving Pregnancy Rates in Subfertile Women Without Other Gynaecological Symptoms: A Systematic Review. Hum Reprod Update (2010) 16(1):1-11. doi: 10.1093/humupd/ dmp033

8. El-Toukhy T, Sunkara SK, Coomarasamy A, Grace J, Khalaf Y. Outpatient Hysteroscopy and Subsequent IVF Cycle Outcome: A Systematic Review and

\section{DATA AVAILABILITY STATEMENT}

The raw data supporting the conclusions of this article will be made available by the authors, without undue reservation.

\section{ETHICS STATEMENT}

The studies involving human participants were reviewed and approved by The Ethics committee of Tehran University of Medical Sciences- IR.TUMS.REC.1394.1598. The patients/ participants provided their written informed consent to participate in this study.

\section{AUTHOR CONTRIBUTIONS}

AA: designing the study, patient selection, supervising the study, revising the manuscript. HF: helping in designing the study and revising the manuscript. FG: helping in preparing the draft, helping in data analysis. MS: doing statistical analysis, revising the manuscript. MG: designing the study, operating the procedures, data gathering,preparing the draft, revising the manuscript. All the authors have read, revised and confirmed the final version of the manuscript.

\section{ACKNOWLEDGMENTS}

We would like to thank all the participants in this study.

Meta-Analysis. Reprod BioMed Online (2008) 16(5):712-9. doi: 10.1016 S1472-6483(10)60486-5

9. Pundir J, Pundir V, Omanwa K, Khalaf Y, El-Toukhy T. Hysteroscopy Prior to the First IVF Cycle: A Systematic Review and Meta-Analysis. Reprod BioMed Online (2014) 28(2):151-61. doi: 10.1016/j.rbmo.2013.09.025

10. Elsetohy KA, Askalany AH, Hassan M, Dawood Z. Routine Office Hysteroscopy Prior to ICSI vs. ICSI Alone in Patients With Normal Transvaginal Ultrasound: A Randomized Controlled Trial. Arch Gynecol Obstet (2015) 291(1):193-9. doi: 10.1007/s00404-014-3397-z

11. Takahashi K, Mukaida T, Tomiyama T, Oka C. High Pregnancy Rate After Hysteroscopy With Irrigation in Uterine Cavity Prior to Blastocyst Transfer in Patients Who Have Failed to Conceive After Blastocyst Transfer. Fertil Steril (2000) 74(3):S206. doi: 10.1016/S0015-0282(00)01328-5

12. Yu HT, Weyers S, Puttemans P, Panayotidis C, Van Herendael B, Gomel V. The Role of Diagnostic Hysteroscopy Before the First In Vitro Fertilization/ Intracytoplasmic Sperm Injection Cycle. Arch Gynecol Obstet (2012) 286 (5):1323-8. doi: 10.1007/s00404-012-2462-8

13. Giorgetti C, Terriou P, Auquier P, Hans E, Spach JL, Salzmann J, et al. Embryo Score to Predict Implantation After in-Vitro Fertilization: Based on 957 Single Embryo Transfers. Hum Reprod (1995) 10(9):2427-31.doi: 10.1093/ oxfordjournals.humrep.a136312

14. De los Santos MJ, Apter S, Coticchio G, Debrock S, Lundin K, Plancha CE, et al. Revised Guidelines for Good Practice in IVF Laboratories, (2015). Hum Reprod (2016) 31(4):685-6. doi: 10.1093/humrep/dew016

15. Rama Raju GA, Shashi Kumari G, Krishna KM, Prakash GJ, Madan K. Assessment of Uterine Cavity by Hysteroscopy in Assisted Reproduction Programme and its Influence on Pregnancy Outcome. Arch Gynecol Obstet (2006) 274(3):160-4. doi: 10.1007/s00404-006-0174-7

16. van Hoogenhuijze NE, Kasius JC, Broekmans FJM, Bosteels J, Torrance HL. Endometrial Scratching Prior to IVF; Does it Help and for Whom? A 
Systematic Review and Meta-Analysis. Hum Reprod Open (2019) 2019(1): hoy025. doi: 10.1093/hropen/hoy025

17. van Hoogenhuijze NE, Mol F, Laven JSE, Groenewoud ER, Traas MAF, Janssen CAH. Endometrial Scratching in Women With One Failed IVF/ICSI Cycle-Outcomes of a Randomised Controlled Trial (SCRaTCH). Hum Reprod (2021) 36(1):87-98. doi: 10.1093/humrep/deaa268

18. Karimzade MA, Oskouian H, Ahmadi S, Oskouian L. Local Injury to the Endometrium on the Day of Oocyte Retrieval has a Negative Impact on Implantation in Assisted Reproductive Cycles: A Randomized Controlled Trial. Arch Gynecol Obstet (2010) 281(3):499-503. doi: 10.1007/s00404-009-1166-1

19. Maheux-Lacroix S, Dodin S, Moore L, Bujold E, Lefebvre J, Bergeron MÈ. Preovulatory Uterine Flushing With Saline as a Treatment for Unexplained Infertility: A Randomised Controlled Trial Protocol. BMJ Open (2016) 6(1): e009897. doi: 10.1136/bmjopen-2015-009897

20. Di Spiezio Sardo A, Di Carlo C, Minozzi S, Spinelli M, Pistotti V, Alviggi C. Efficacy of Hysteroscopy in Improving Reproductive Outcomes of Infertile Couples: A Systematic Review and Meta-Analysis. Hum Reprod Update (2016) 22(4):479-96. doi: 10.1093/humupd/dmw008

21. Potdar N, Gelbaya T, Nardo LG. Endometrial Injury to Overcome Recurrent Embryo Implantation Failure: A Systematic Review and Meta-Analysis. Reprod BioMed Online (2012) 25(6):561-71. doi: 10.1016/j.rbmo.2012.08.005

22. Inagaki N, Stern C, McBain J, Lopata A, Kornman L, Wilkinson D. Analysis of Intra-Uterine Cytokine Concentration and Matrix-Metalloproteinase Activity in Women With Recurrent Failed Embryo Transfer. Hum Reprod (2003) 18(3):60815. doi: 10.1093/humrep/deg139

23. Johnson NP, Bhattu S, Wagner A, Blake DA, Chamley LW. Lipiodol Alters Murine Uterine Dendritic Cell Populations: A Potential Mechanism for the Fertility-Enhancing Effect of Lipiodol. Fertil Steril (2005) 83(6):1814-21. doi: 10.1016/j.fertnstert.2004.11.065

24. Johnson NP, Farquhar CM, Hadden WE, Suckling J, Yu Y, Sadler L. The FLUSH Trial-Flushing With Lipiodol for Unexplained (and EndometriosisRelated) Subfertility by Hysterosalpingography: A Randomized Trial. Hum Reprod (2004) 19(9):2043-51. doi: 10.1093/humrep/deh418
25. Lier MCI, Özcan H, Schreurs AMF, van de Ven PM, Dreyer K, van der Houwen LEE, et al. Uterine Bathing With Sonography Gel Prior to IVF/ICSITreatment in Patients With Endometriosis, a Multicentre Randomised Controlled Trial. Hum Reprod Open (2020) 2020(4):hoaa054. doi: 10.1093/ hropen/hoaa054

26. Reilly SJ, Glanville EJ, Dhorepatil B, Prentice LR, Mol BW, Johnson NP. The IVF-LUBE Trial - a Randomized Trial to Assess Lipiodol $\left({ }^{\circledR}\right)$ Uterine Bathing Effect in Women With Endometriosis or Repeat Implantation Failure Undergoing IVF. Reprod BioMed Online (2019) 38(3):380-6. doi: 10.1016/ j.rbmo.2018.11.015

27. Salehpour S, Zamaniyan M, Saharkhiz N, Zadeh Modares S, Hosieni S, Seif S. Does Intrauterine Saline Infusion by Intrauterine Insemination (IUI) Catheter as Endometrial Injury During IVF Cycles Improve Pregnancy Outcomes Among Patients With Recurrent Implantation Failure?: An RCT. Int J Reprod BioMed (2016) 14(9):583-8. doi: 10.29252/ijrm.14.9.583

Conflict of Interest: The authors declare that the research was conducted in the absence of any commercial or financial relationships that could be construed as a potential conflict of interest.

Publisher's Note: All claims expressed in this article are solely those of the authors and do not necessarily represent those of their affiliated organizations, or those of the publisher, the editors and the reviewers. Any product that may be evaluated in this article, or claim that may be made by its manufacturer, is not guaranteed or endorsed by the publisher.

Copyright $\odot 2022$ Ghasemi, Aleyasin, Fatemi, Ghaemdoust and Shahrakipour. This is an open-access article distributed under the terms of the Creative Commons Attribution License (CC BY). The use, distribution or reproduction in other forums is permitted, provided the original author(s) and the copyright owner(s) are credited and that the original publication in this journal is cited, in accordance with accepted academic practice. No use, distribution or reproduction is permitted which does not comply with these terms. 